\title{
Hepatoprotective and antiapoptotic role of aged black garlic against hepatotoxicity induced by cyclophosphamide
}

Rania A. Ahmed

\begin{abstract}
Background: Cyclophosphamide (CP) is an anticancer drug utilized for treatment of many types of cancer. CP causes many side effects such as hepatotoxicity and mutagenicity. Garlic has long been used as a spice and food additive and is considered as one of the important medicinal plants. Garlic had medicinal importance as it has hepatoprotective, antimicrobial, antioxidant, anti-inflammatory, antithrombotic, antihypertensive, and antihyperlipidemic properties. The purpose of the presented study is to investigate the protective role of aged black garlic against cyclophosphamide-induced hepatotoxicity and apoptosis. The present work was carried out by routine histological H\&E stain, histochemical Feulgen stain for DNA, immunohistochemical stain Bcl-2 as apoptotic marker, comet assay, serum biochemical analysis for liver function markers (ALT, AST, albumin, and bilirubin), and hepatic oxidative stress markers (MDA, CAT, SOD, and GPX).
\end{abstract}

Results: Cyclophosphamide induced histological alteration as fatty degeneration, blood venous congestion, pyknosis, necrosis, and vaculations. CP caused a decrease of DNA content, an increase of BCl-2 immunoreactivity, and increase of DNA damage. Biochemical investigations showed that CP induced significant elevation of ALT, AST, bilirubin, and hepatic MDA. Moreover, CP induced reduction of albumin and hepatic SOD, CAT, and GPx values. Aged black garlic induced significant improvement of alterations induced by CP, when compared with CP-treated group.

Conclusion: Aged black garlic succeeded in protecting the liver from apoptosis and pathological alterations induced by CP.

Keywords: Aged black garlic, Apoptosis, Bcl-2, Comet assay, Cyclophosphamide, DNA damage, Histology, Liver

\section{Background}

Liver injury caused by toxic chemicals and certain drugs, especially anticancer drugs, is a common toxicological problem. The damage pathogenesis is ranging from inflammation to apoptosis (El-Halawany, Salah El Dine, El Sayed, \& Hattori, 2014).

Application of anticancer drugs causes toxic impacts on healthy cells alongside on tumor cells due to its toxic metabolites. Cyclophosphamide $(\mathrm{CP})$ is an anticancer drug utilized for treatment of many types of cancer. Therapeutic dose of CP causes hepatotoxicity, nephrotoxicity, cardiotoxicity, immunotoxicity, and mutagenicity (Chamorro-Cevallos et al., 2008; Viswanatha-Swamy et al., 2013; Amien, Fahmy, Abd-Elgleel, \& Elaskalany, 2015; Li, Li, \& Jia, 2015). Metabolism of CP leads to the

Correspondence: Rania.sheir@yahoo.com

Zoology Department, Faculty of Science, Suez University, Suez, Egypt formation of many cytotoxic metabolites that induce oxidative injury (Olayinka, Ore, Ola, \& Adeyemo, 2015). The vital issue for both therapeutic and toxic effects of $\mathrm{CP}$ is the requirement of metabolic activation by hepatic microsomal cytochrome P450 mixed functional oxidase system (Cederbaum, 2015). Consequently, the mitigation of the liver damage caused by $\mathrm{CP}$ could be an important clinical downside to be resolved. The pharmacodynamic constituents from natural herbs in complementary and alternative medicine (CAM) have been thought to be the main target among the combat with $\mathrm{CP}$-induced liver damage (Yin, Wei, Jian, \& Yang, 2013).

Garlic (Allium sativum L. family Liliaceae) has long been used as a spice and food additive and is considered as one of the important medicinal plants and provides a useful source of new therapeutics. Garlic has a great pharmacological role, as it has been found that the sulfur-containing compounds of garlic have antimutagenic and 
anticarcinogenic effects (Mikaili, Maadirad, Moloudizargari, Aghajanshakeri, \& Sarahroodi, 2013; Kimura et al., 2017).

Aged black garlic (ABG) is a garlic-processed product which is prepared by aging raw garlic through thermal process at high temperature and high humidity. Also, it is available on the markets in the form of whole bulbs, powder, or organic capsules (Kim et al., 2011). ABG was found to show more antioxidant activity and higher content of total polyphenol than fresh garlic (Choi et al., 2008; Shin, Choi, Lee, Cha, \& Sung, 2008; Jeong et al., 2016). Aged black garlic also has been largely attributed to as having cardioprotective, antidiabetic, hepatoprotective, antimicrobial, antioxidant, anti-inflammatory, antithrombotic, antihypertensive, and antihyperlipidemic properties. (Shaarawy et al., 2009; Obioha, Suru, OlaMudathir, \& Faremi, 2009; García-Villalón et al., 2016; Kim et al., 2017; Kimura et al., 2017).

The present study was aimed to investigate the possible protective effects of aged black garlic on histopathological, immunohistochemical, genotoxic, and biochemical alterations induced by cyclophosphamide in the liver of albino rats.

\section{Methods}

\section{Animals}

Twenty-eight adult male Sprague-Dawley rats weighing $180 \pm 15 \mathrm{~g}$ were included in the present investigation. The animals were obtained from the Animal House, National Research Center (NRC), Giza, Egypt, and housed in plastic cages (seven rats/cage) at normal atmospheric temperature $\left(25 \pm 2{ }^{\circ} \mathrm{C}\right)$ and normal 12-h light/dark cycle. Rodent diet and water were provided ad libitum. For acclimatization and to exclude any infection, rats were kept under observation for 1 week prior to experimentation.

\section{Chemicals}

\section{Cyclophosphamide}

$\mathrm{CP}$ was obtained in the form of powder (Endoxan injection vial) manufactured by Baxter Oncology, Germany.

\section{Aged black garlic}

Aged black garlic, $1 \mathrm{~g}$ organic capsules, were obtained from (Mercola Health Resources, LLC; USA).

\section{Experimental design}

Rats were randomly divided into four groups $(n=7)$.

Group 1: This group was kept as control, without any treatments.

Group 2: Animals of this group received aged black garlic (ABG) at dose of ( $200 \mathrm{mg} / \mathrm{kg} /$ daily orally for 2 weeks).

Group 3: The animals of this group were left for 2 weeks without any treatment, then received a single dose of cyclophosphamide ( $200 \mathrm{mg} / \mathrm{kg}$, i.p.).
Group 4: Animals of this group were treated with ABG for 2 weeks, then received a single dose of cyclophosphamide (200 mg/kg, i.p.).

\section{Methods}

\section{Histopathological and histochemical examinations}

At the end of the experiment, animals were sacrificed by cervical dislocation, then dissected out and their livers were removed, and then fixed in $10 \%$ neutral formalin, dehydrated, cleared, and embedded in paraffin wax.

a. For histological examination, paraffin sections of $5 \mu \mathrm{m}$ thickness were prepared and stained with routine hematoxylin and eosin stain (Drury \& Wallington, 1980).

b. Feulgen reaction (Bancroft \& Gamble, 2002) was applied for the histochemical demonstration of nuclear DNA.

\section{Immunohistochemical examination}

For immunohistochemical localization of $\mathrm{Bcl}-2$, which is a pro-apoptotic protein, paraffin sections with $5 \mu \mathrm{m}$ thickness sections were stained using the avidin-biotin peroxidase method. Slides were incubated with primary mouse anti-Bcl-2 (diluted 1:200; Santa Cruz Biotechnology, Santa Cruz, CA, USA), for $60 \mathrm{~min}$. Then, slides were incubated with a biotinylated secondary antibody for $15 \mathrm{~min}$, then incubated with horseradish peroxidase conjugated with streptavidin for further $15 \mathrm{~min}$, followed by adding 3-amino-9-ethylcarbasole (Dako Cytomation, USA) for $15 \mathrm{~min}$. The positive sites of the anti-Bcl-2 antibodies were stained with brown color (Pedrycz \& Czerny, 2008).

\section{Comet assay}

This technique permits the detection and an evaluation of single-stranded DNA breaks. The alkaline comet assay was performed as described by Singh, Muller, and Berger (2003).

\section{Biochemical studies Serum biochemical analysis}

Blood samples were collected from the retro-orbital venous plexus before sacrificing animals by using clean capillary tubes. Blood samples were left to clot at room temperature and then centrifuged at $3000 \mathrm{rpm}$ for $15 \mathrm{~min}$ to separate serum. Sera were stored at $20{ }^{\circ} \mathrm{C}$ until assayed for the biochemical parameters. Serum aspartate aminotransferase (AST), alanine aminotransferase (ALT), albumin, and bilirubin were assayed according to the method of Schumann and Klauke (2003) using reagent kit purchased from Biosystems (Spain). 
Hepatic tissue biochemical analysis for antioxidant markers For antioxidant demonstration in hepatic tissue, clear supernatants were separated and used for analysis:

1. The extent of lipid peroxidation was estimated as the concentration of thiobarbituric acid reactive product (malondialdehyde (MDA)) according to Ohkawa, Ohishi, and Yagi (1979).

2. Superoxide dismutase (SOD) activity was measured using the methods of Rest and Spitznagel (1977).

3. Catalase (CAT) activity was determined from the rate of decomposition of H2O2 (Aebi, Wyss, Scherz, \& Skvaril, 1974).

4. Glutathione peroxidase (GPx) was measured using the methods of Rotruck et al. (1973).

\section{Statistical analysis}

Statistical analysis was performed using SPSS v.16. Results were articulated as mean \pm standard error (SE), and all statistical comparisons were made by means of one- way ANOVA test followed by Duncan's multiple range test post hoc analysis.

A $P$ value $<0.05$ was considered significant.

\section{Results}

Histological results

Microscopic examination of hepatic parenchyma of both control group and aged black garlic (ABG)-treated group exhibited normal lobular architecture with hepatocytes arranged in hepatic cords radiating around the central vein and separated by obvious blood sinusoids. The sinusoidal endothelium is formed of endothelial lining cells and contains phagocytic Kupffer cells. The individual hepatocyte was polyhedral in shape with central round nucleus, and some hepatocytes were binucleated (Fig. 1a). Cyclophosphamide induced many histopathological alterations when compared with the control group, such as leucocytic infiltration (Fig. 1b), blood venous congestion, cytoplasmic vaculation, and interlobular hemorrhage. Some hepatocytes were pyknotic
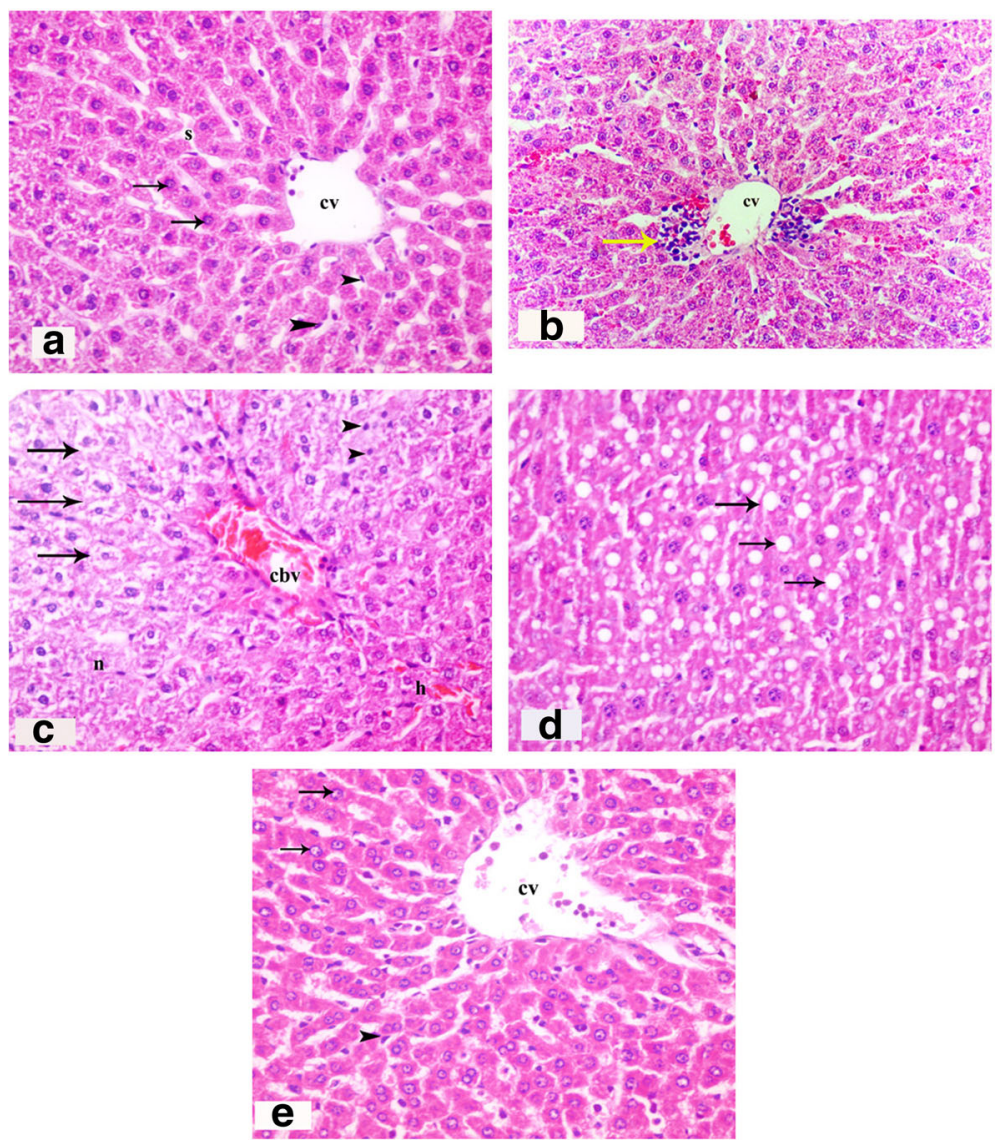

Fig. 1 a Section in control rat showing classic hepatic architecture with normal central vein (cv), hexagonal hepatocyte with central round nucleus (arrow), sinusoids (s) with Kupffer cells (arrow head). b Section in rat treated with CP showing leucocytic infiltration (yellow arrow). c CP-treated rat showing congested blood vessel (cbv), cytoplasmic vaculation (arrows), necrosis (n), pyknosis (arrow head), hemorrhage (h). d CP-treated rat showing fatty degeneration. e Section in rat treated with $\mathrm{ABG}$ and CP showing marked improvement of hepatic tissue with normal hepatocytes (arrows) radiating around central vein $(\mathrm{CV}) .(\mathrm{H} \& \mathrm{E}, \times 400)$ 
while others exhibited necrosis. (Fig. 1c). Fatty degeneration was observed markedly (Fig. 1d).

On the other hand, the examination of rats treated with $\mathrm{ABG}$ before $\mathrm{CP}$ showed marked improvement of hepatic tissues when compared with $\mathrm{CP}$-treated group. Hepatocytes were rearranged in radiating strands, nuclei were normal, and cytoplasm did not show any vacuoles or fat droplets (Fig. 1e).

\section{Histochemical results}

For demonstration of DNA content in nuclei, sections were stained with Feulgen's reaction to demonstrate DNA as magenta color. Sections of the liver of control and ABG-treated groups have shown normal DNA contents (Fig. 2a). Cyclophosphamide caused marked reduction number of nuclei and in DNA content which was explained by moderate magenta color in the hepatocytes of liver sections (Fig. 2b).

Treating rats with $\mathrm{ABG}$ and $\mathrm{CP}$ showed more obvious improvement in DNA content with increasing number of nuclei when compared with CP-treated group (Fig. 2c).

\section{Immunohistochemical results}

Microscopic examination of both control and ABG-treated groups revealed moderate expression of the antiapoptotic protein, $\mathrm{Bcl}-2$ in hepatocyte cytoplasm (Fig. 3a). Treating rats with CP caused marked increased expression of BCL-2 in hepatocytes (Fig. 3b).

On the other hand, animals treated with $\mathrm{ABG}$ and $\mathrm{CP}$ showed weak expression of BCL-2 when compared with the CP-treated group (Fig. 3c).

\section{Comet assay results}

Data presented in Table 1 and Fig. 4 showed the genotoxic effect of $\mathrm{CP}$ on hepatocytes and the protective role of ABG. The genotoxic effect was observed as a significant increase $(P<0.05)$ in the percentage of DNA in the comet tail in the nuclei of hepatocytes.

Treatment with ABG did not cause any significant increase of DNA fragmentation when compared with the control group (Table 1; Fig. 4a). CP administration caused a significant increase $(P<0.05)$ in number of tailed nuclei and in DNA strand breaks which lead to greater DNA migration out of the nucleus into the tail of the comet in the hepatocytes when compared to the control group (Table 1; Fig. 4b).

Rats treated with ABG then $\mathrm{CP}$ showed significant improvement in DNA by decreasing the number of damaged and strongly damaged spots when compared with the CP-treated group (Table 1; Fig. 4c).

\section{Biochemical results}

The effects of CP-induced hepatotoxicity and the protective effects of $A B G$ on serum markers of liver function are shown in Table 2. Treating rats with ABG did not cause any alterations of liver function marker levels when compared with the control group.

Cyclophosphamide induced significant elevation $(P<0.05)$ of serum activity of the liver function markers (ALT, AST, and bilirubin). Conversely, albumin was significantly decreased in CP-administered rats compared with control rats. On the other hand, oral administration of ABG before CP significantly decreased ALT, AST, and bilirubin, while albumin was increased when compared with the CP-treated group (Table 2).

\section{The oxidative stress markers in hepatic tissue}

Data in Table 3 showed that the ABG group did not cause any significant alterations in hepatic oxidative stress markers when compared to the control group. Cyclophosphamide administration induced significant reduction $(P<0.05)$ of hepatic antioxidant enzymes (CAT, SOD, and GPx) values, while MDA values were significantly increased $(P<0.05)$ (Table 3$)$.

In comparison with the CP-treated group, treating animals with $\mathrm{ABG}$ then $\mathrm{CP}$ induced significant elevation $(P<0.05)$ of CAT, SOD, and GPx. Conversely, the activity of MDA significantly decreased $(P<0.05)$ (Table 3).

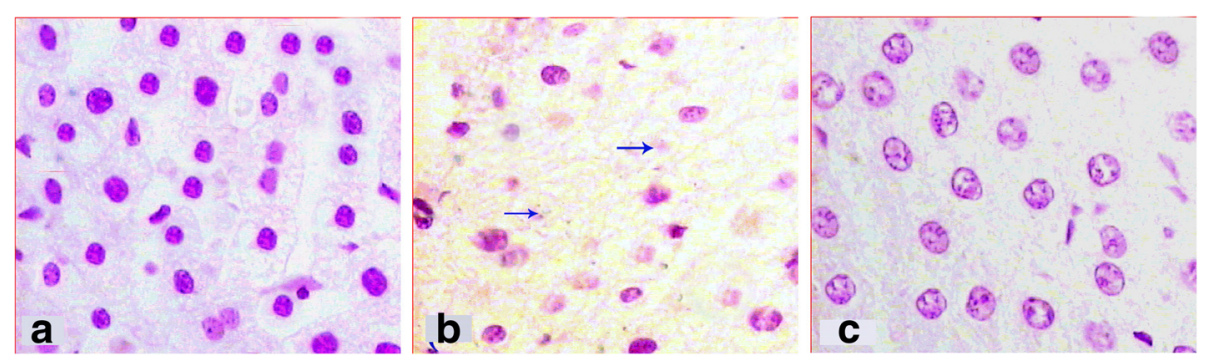

Fig. 2 a Section in control rat showing normal content of DNA in nuclei of hepatocytes. $\mathbf{b}$ CP-treated rat showing marked reduction of DNA content in nuclei of hepatocytes with decreasing number of nuclei (arrows). c Sections in rat treated with ABG + CP showing marked improvement of DNA content with increasing number of nuclei (Feulgen histochemical stain, $\times 400$ ) 


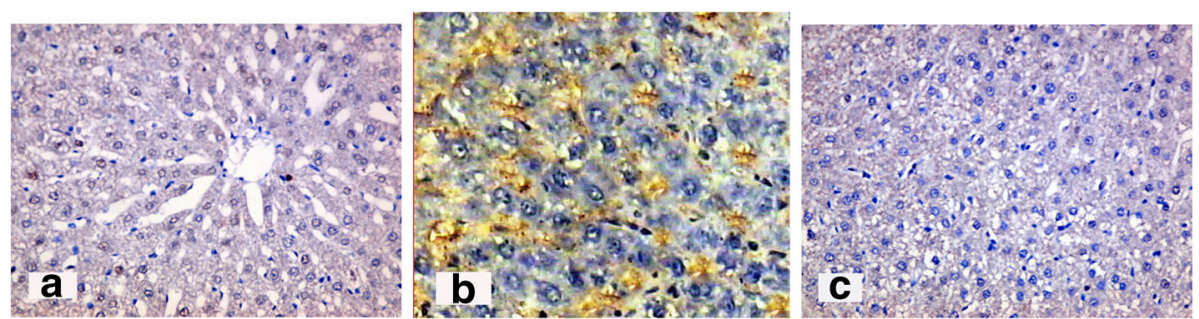

Fig. 3 a Section of control rat showing no expression of $\mathrm{BCL}-2$. $\mathbf{b}$ CP-treated rat showing a marked increase in $\mathrm{BC}-2$ immunoreactivity in the hepatocytes (brown color). c Rat treating with ABG then CP showing weak expression in Bcl-2 immunostaining (BCl-2 immunostain, $\times 400$ )

\section{Discussion}

The presented work showed that cyclophosphamide induced many histopathological alterations when compared with the control group. Liver sections of CP-treated rats showed leucocytic infiltration, blood venous congestion, cytoplasmic vaculation, interlobular hemorrhage, fatty degeneration, and necrosis. In addition, some hepatocytes were pyknotic while others were karyolitic.

Many studies recorded the cyclophosphamide induced hepatic histopathological changes. $\mathrm{CP}$ was proven to cause infiltration of acute inflammatory cells and congestion of blood vessels, focal necrosis, pyknosis, and karyolysis. In addition, CP causes focal degeneration of hepatocytes, hydropic degeneration, fatty degeneration, cytoplasmic vaculations, hyperproliferation around the deformed bile ducts, dysplasia, karyomegaly, and nuclear atypia (Lata, Singh, NathTiwari, \& Upadhyay, 2014; Mahmoud \& Al Dera, 2015; Li, Li, \& Jia, 2015). Metabolism of cyclophosphamide occurs by the hepatic cytochrome $\mathrm{P} 450$ system, which results in sinusoidal obstruction syndrome, which induces necrosis, obstruction, and congestion of hepatic veins ( $\mathrm{Li}, \mathrm{Li}, \&$ Jia, 2015).

Our data further suggests that examination of rats treated with aged black garlic before $\mathrm{CP}$ showed marked improvement of hepatic tissues when compared with the $\mathrm{CP}$-treated group. Hepatocytes were rearranged in radiating strands, most of nuclei were normal, and cytoplasm did not show any vacuoles or fat drops. The blood venous congestion and necrosis were reduced to a less degree, with a less infiltration in the hepatic portal area and less cellular degeneration.

There exist some limited studies that have surveyed on the histological effects of aged black garlic on the liver. Shin et al. (2014) recorded that aged black garlic ameliorated the CCl4-induced histopathological alterations in hepatocytes, such as infiltration of immune cells and necrosis. Moreover, aged black garlic treatment markedly suppressed the accumulation of lipids and decreased fatty degeneration in mice.

Concerning DNA damage and apoptosis, this study recorded that histochemical demonstration of nuclear DNA by Feulgen stain showed that cyclophosphamide caused marked reduction number of nuclei and in their DNA content. Moreover, immunohistochemical demonstration of antiapoptotic protein BCL-2 showed that treating rats with $\mathrm{CP}$ caused marked increased expression of BCL-2 in hepatocytes.

Furthermore, the results obtained from DNA damage investigated by comet assay revealed that $\mathrm{CP}$ administration caused marked degree of DNA damage as there was a significant increase number $(P<0.05)$ of tailed nuclei, and in DNA strand breaks which lead to greater DNA migration out of the nucleus into the tail of the comet in the hepatocytes when compared to the control group. Many investigations have reported that $\mathrm{CP}$ induces gene mutations, DNA strand breaks, chromosome aberrations, micronuclei and sister chromatid exchanges, apoptosis, and generation of free radicals (Ferreira et al., 2013).

Table 1 Ameliorative effect of aged black garlic (ABG) on the migration of nuclear DNA in hepatocytes of the cyclophosphamide (CP)-treated rats

\begin{tabular}{llllll}
\hline Groups & Parameters & & & & \\
\cline { 2 - 6 } & Tailed\% & Untailed\% & Tail length $(\mu \mathrm{m})$ & Tail DNA\% & Tail moment \\
\hline Control & $5.00 \pm 1.00$ & $95.00 \pm 1.00$ & $1.95 \pm 1.0001$ & $1.670 \pm 1.00$ & $3.30 \pm 1.091$ \\
ABG & $6.00 \pm 1.00$ & $94.00 \pm 1.00$ & $1.90 \pm 1.0001$ & $1.72 \pm 1.00$ & $3.30 \pm 1.091$ \\
CP & $33.13 \pm 0.808^{*}$ & $66.87 \pm 0.808^{*}$ & $3.89 \pm 0.105^{*}$ & $3.703 \pm 0.391^{*}$ & $14.257 \pm 1.527^{*}$ \\
ABG + CP & $8.10 \pm 1.015^{* *}$ & $91.90 \pm 1.015^{* *}$ & $2.73 \pm 1.0017^{* *}$ & $2.707 \pm 1.006^{* *}$ & $7.317 \pm 1.0001^{* *}$ \\
\hline
\end{tabular}

Data are represented as mean $\pm \mathrm{SD}, n=7$

* Significant increase at $P<0.05$ compared with the control group

**Significant decrease at $P<0.05$ compared with the cyclophosphamide group 

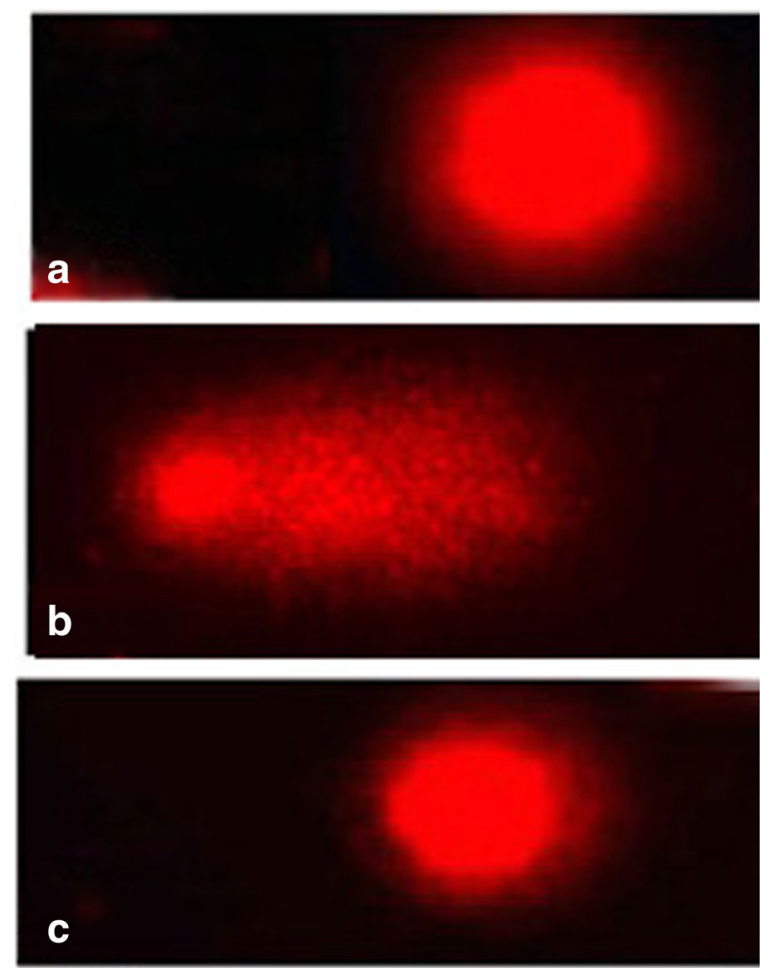

Fig. 4 Fluorescent microscope photomicrographs of hepatic cells. a Normal DNA spots (no migration). b CP-treated rat shows strong damaged DNA spots (migration towards the anode). c Hepatocyte of rat treated with $A B G+C P$ showing improvement in DNA and reduction of damaged spots (bromide ethidium staining, $\times 400$ )

CP-induced hepatocytes apoptosis was investigated by Alqahtani and Mahmoud (2016) as they determined immunohistochemically both gene and protein expression levels of the proapoptotic factors caspase-3 and BAX. Their results showed that the liver of CP-treated rats for 2 weeks revealed that $\mathrm{CP}$ induced significant increase of both caspase-3 mRNA expression $(P<0.01)$ and protein levels $(P<0.05)$.

Table 2 Ameliorative effect of aged black garlic (ABG) on the level of some serum biochemical parameters of the cyclophosphamide (CP)-treated rats

\begin{tabular}{|c|c|c|c|c|}
\hline \multirow[t]{2}{*}{ Groups } & \multicolumn{4}{|l|}{ Parameters } \\
\hline & $\overline{A L T}(\mathrm{U} / \mathrm{L})$ & AST (U/L) & Alb. (g/dL) & Bil. (mg/dL) \\
\hline Control & $24.6 \pm 4.1$ & $33.4 \pm 4.8$ & $4.0 \pm 0.13$ & $0.34 \pm 0.03$ \\
\hline$A B G$ & $25.6 \pm 4.3$ & $36.8 \pm 4.87$ & $3.9 \pm 0.13$ & $0.37 \pm 0.02$ \\
\hline $\mathrm{CP}$ & $88.8 \pm 6.34^{*}$ & $106.0 \pm 7.5^{*}$ & $2.0 \pm 0.16^{*}$ & $0.51 \pm 0.03^{*}$ \\
\hline$A B G+C P$ & $34.6 \pm 23.62^{* *}$ & $48.4 \pm 2.0^{* *}$ & $3.4 \pm 0.19^{* *}$ & $0.40 \pm 0.04^{* *}$ \\
\hline
\end{tabular}

Data are represented as mean $\pm \mathrm{SD}, n=7$

* Significant increase at $P<0.05$ compared with the control group

**Significant decrease at $P<0.05$ compared with the cyclophosphamide group
Zhang et al. (2012) proved that the CP produced significant DNA damage and cell apoptosis in mouse bone marrow cells and peripheral blood leukocytes, DNA damage, and cell apoptosis were investigated by single cell gel electrophoresis, flow cytometry assay with annexin V-FITC/propidine iodide (PI), and acridine orange (AO)/ethidium bromide (EB) staining assay.

Gore et al. (2016) investigated the effect of $\mathrm{CP}$ on bladder genomic DNA by agarose gel electrophoresis in mice, and they found that genomic DNA samples isolated from bladders of mice treated with cyclophosphamide showed significantly greater DNA fragmentation. Cell apoptosis is involved in the CP-induced acute hepatotoxicity, characterized by activation of immunohistochemical marker caspase-3, a key cell death protease (Li et al., 2015).

We demonstrated here that $A B G$ is able to prevent the genotoxicity induced by cyclophosphamide. Histochemical observations using Feulgen stain revealed that treating rats with $\mathrm{ABG}$ and $\mathrm{CP}$ showed more obvious improvement in DNA content with increasing number of nuclei when compared with the $\mathrm{CP}$-treated group. Moreover, immunohistochemical stain $\mathrm{Bcl} 2$ results showed that treating animals with $\mathrm{ABG}$ and $\mathrm{CP}$ caused a weak expression of $\mathrm{Bcl}-2$ when compared with the $\mathrm{CP}$ treated group. Concerning DNA damage, comet assay recorded that rats treated with $\mathrm{ABG}$ then $\mathrm{CP}$ showed significant improvement in DNA by decreasing the number of damaged and strongly damaged spots when compared with the CP-treated group.

Our results are supported by the previous investigation of Abdella and Gad (2008), as they proved that the DADS (diallyl disulfide compound), as the most important biological active compound of garlic, revealed a significant protective effect against mutagenic, genotoxic, and DNA strand breaks and chromosomal damage induced by the mercuric chloride. In addition, Abdella and Gad (2008) explained the role of garlic as antigenotoxic agent may be due to the presence of sulfhydryl groups which may provide protection against increasing free radical formation which induce DNA damage.

Currently, histopathological and genotoxic results were supported and confirmed by biochemical results. Cyclophosphamide induced significant elevation $(P<0.05)$ of serum activity of the liver function markers (ALT, AST, and bilirubin). Conversely, albumin was significantly decreased in CP-administered rats compared with control rats. Similar findings were reported by many authors, as cyclophosphamide was proved to cause significant increase of serum ALT, AST, and bilirubin (Olayinka, Ore, Ola, \& Adeyemo, 2015; Li et al., 2015) and decreased albumin levels (Mahmoud \& Al Dera, 2015).

Our results revealed that oral administration of $A B G$ before CP significantly decreased ALT, AST, and 
Table 3 Ameliorative effect of aged black garlic (ABG) on some oxidative stress parameters of the cyclophosphamide (CP)-treated rats

\begin{tabular}{lllll}
\hline Groups & \multicolumn{3}{l}{} & \\
\cline { 2 - 5 } & MDA (nmol/g wet tissue) & CAT (U/g tissue) & SOD (U/mg tissue) & GPx (IU/g wet tissue) \\
\hline Control & $51.7 \pm 1.3$ & $9.44 \pm 0.45$ & $3.70 \pm 0.07$ & $48.80 \pm 2.65$ \\
ABG & $54.1 \pm 2.6$ & $9.12 \pm 0.25$ & $3.30 \pm 0.25$ & $50.06 \pm 4.12$ \\
CP & $77.8 \pm 1.8^{*}$ & $5.54 \pm 0.30^{*}$ & $2.40 \pm 0.15^{*}$ & $24.22 \pm 2.34^{*}$ \\
ABG + CP & $63.1 \pm 1.6^{* *}$ & $7.62 \pm 0.29^{* *}$ & $3.12 \pm 0.14^{* *}$ & $42.44 \pm 3.63^{* *}$ \\
\hline
\end{tabular}

Data are represented as mean $\pm \mathrm{SD}, n=7$

*Significant increase at $P<0.05$ compared with the control group

**Significant decrease at $P<0.05$ compared with the cyclophosphamide group

bilirubin, while albumin was increased when compared with the CP-treated group. Similar findings were recorded; ABG significantly decreased ALT, AST, and ALP serum levels which were elevated by $\mathrm{CCl} 4$ administration (Shin et al., 2014).

Concerning the oxidative stress and antioxidant statues, our investigation showed that cyclophosphamide administration induced significant reduction $(P<0.05)$ of hepatic antioxidant enzymes (CAT, SOD, and GPx) values, while MDA values were significantly increased $(P<0.05)$. Many investigations have reported that $\mathrm{CP}$ induces oxidative stress and reduces the antioxidant statues. Administration with $\mathrm{CP}$ was proven to cause a significant reduction of hepatic CAT, SOD, and GPx (Mahmoud, Hussein, \& Ramadan, 2013; Mahmoud \& Al Dera, 2015; Olayinka, Ore, Ola, \& Adeyemo, 2015). Moreover, liver lipid peroxidation (LPO), estimated as MDA, showed a significant increase $(P<0.001)$ in CP-induced mice when compared to the control group (Li et al., 2015).

The present results showed that supplementation of ABG before the administration of CP induced significant elevation $(P<0.05)$ of CAT, SOD, and GPx. Conversely, the activity of MDA significantly decreased $(P<0.05)$. Similarly, Shaarawy et al. (2009) recorded that administration of garlic significantly decreased lipid peroxidation and increased antioxidant enzymes, such as SOD, CAT, and GSH significantly.

The most active compound in black garlic is S-allyl-Lcysteine (SAC) which can act as an antioxidant via two pathways, direct and in direct pathway. Directly, SAC scavenges free radicals. Indirectly, SAC restores glutathione peroxidase, glutathione reductase, and superoxide dismutase levels and activates the transcription Nrf2 factor, which is responsible for regulating the expression of antioxidant and cellular protective genes by inhibition of $\mathrm{NFjB}$ factor which is activated in response to oxidative stress. (Hermawati, Sari, \& Partadiredja, 2015).

Summarily, CP induces inflammation and oxidative stress in the liver of rats. Oxidative stress together with inflammation induces apoptosis (Alqahtani \& Mahmoud, 2016). CP-induced apoptosis is mediated by oxidative stress (Asiri, 2010). Aged black garlic may act as an antimutagenic and protective agent against DNA damage via its antioxidant properties (Abdella \& Gad, 2008).

\section{Conclusions}

The present study can suggest that aged black garlic has antiapoptotic properties against $\mathrm{CP}$-induced apoptosis by inhibition of oxidative stress.

\section{Funding \\ The author declares no specific funding for this work.}

\section{Ethics approval and consent to participate}

All the animal protocols used were according to the international standard protocols for the use of laboratory animals (PHS, 2015). The study and all procedures were approved by the Animal Care and Bioethics Committee, Menoufia University, Egypt (Approval No. MNSH-179).

\section{Consent for publication}

Not applicable

\section{Competing interests}

The author declares no competing interests.

\section{Publisher's Note}

Springer Nature remains neutral with regard to jurisdictional claims in published maps and institutional affiliations.

Received: 27 May 2017 Accepted: 5 January 2018

Published online: 30 January 2018

\section{References}

Abdella E. and Gad M. 2008. Protective role of diallyl disulphide compound (from garlic extract) against mercuric chloride-Induced genotoxicity and cytotoxicity in albino rats. Iranian Journal of Cancer Prevention; 1(3): 95-109.

Aebi, H., Wyss, S. R., Scherz, B., \& Skvaril, F. (1974). Heterogeneity of erythrocyte catalase II. Isolation and characterization of normal and variant erythrocyte catalase and their subunits. European Journal of Biochemistry, 48(1), 137-145.

Alqahtani, S., \& Mahmoud, A. (2016). Gamma-glutamylcysteine ethyl ester protects against cyclophosphamide-induced liver injury and hematologic alterations via upregulation of PPAR and attenuation of oxidative stress, inflammation, and apoptosis. Oxidative Medicine and Cellular Longevity, 2016(4016209), 14.

Amien, A. I., Fahmy, S. R., Abd-Elgleel, F. M., \& Elaskalany, S. M. (2015). Renoprotective effect of Mangifera indica polysaccharides and silymarin against cyclophosphamide toxicity in rats. The Journal of Basic \& Applied Zoology, 72, 154-162.

Asiri, Y. A. (2010). Probucol attenuates cyclophosphamide induced oxidative apoptosis, p53 and Bax signal expression in rat cardiac tissues. Oxidative Medicine and Cellular Longevity., 3(5), 308-316.

Bancroft, J. D., \& Gamble, M. (2002). Theory and practice of histological techniques, (5th ed., pp. 377-694). London, New York \& Sydney: Churchill Livingstone.

Cederbaum, I. (2015). Molecular mechanisms of the microsomal mixed function oxidases and biological and pathological implications. Redox Biology, 4, 60-73. 
Chamorro-Cevallos, G., Garduño-Siciliano, L., Barrón, B., Madrigal-Bujaidar, E., CruzVega, D. E., \& Pages, N. (2008). Chemoprotective effect of Spirulina (Arthrospira) against cyclophosphamide-induced mutagenicity in mice. Food and Chemical Toxicology, 46(2), 567-574.

Choi, D. J., Lee, S. J., Kang, M. J., Cho, H. S., Sung, N. J., \& Shin, J. H. (2008). Physicochemical characteristics of black garlic (Allium sativum L.) J Korean. Soc. Food Sci. Nutr, 37(4), 465-471.

Drury, R. A., \& Wallington, E. A. (1980). Carleton's Histological Techniques, (5th ed., p. 362). Oxford: Oxford University Press.

El-Halawany, A. M., Salah El Dine, R., El Sayed, N. S., \& Hattori, M. (2014). Protective effect of Aframomum melegueta phenolics against CCl4-induced rat hepatocytes damage; role of apoptosis and pro-inflammatory cytokines inhibition. Scientific Reports, 4, 5880.

Ferreira, S. G., Peliciari-Garcia, R. A., Takahashi-Hyodo, S. A., Rodrigues, A. C., Amaral, F. G., Berra, C. M., ... Cipolla-Neto, J. (2013). Effects of melatonin on DNA damage induced by cyclophosphamide in rats. Brazilian Journal of Medical and Biological Research, 46, 278-286.

García-Villalón, A. L., Amor, S., Monge, L., Fernández, N., Prodanov, M., Muñoz, M., ... Granado, M. (2016). In vitro studies of an aged black garlic extract enriched in S-allylcysteine and polyphenols with cardioprotective effects. Journal of Functional Foods, 27, 189-200.

Gore, P., Prajapati, C., Mahajan, U., Goyal, S., Belemkar, S., Ojha, S., \& Patil, C. (2016). Protective effect of thymoquinone against cyclophosphamide-induced hemorrhagic cystitis through inhibiting DNA damage and upregulation of Nrf2 expression. International Journal of Biological Sciences, 12(8), 944-953.

Hermawati, E., Sari, D., \& Partadiredja, G. (2015). The effects of black garlic ethanol extract on the spatial memory and estimated total number of pyramidal cells of the hippocampus of monosodium glutamate-exposed adolescent male Wistar rats. Anatomical Science International, 90, 275-286.

Jeong, Y. Y., Ryu, J. H., Shin, J., Kang, M. J., Kang, J. R., Han, J., \& Kang, D. (2016). Comparison of anti-oxidant and anti-inflammatory effects between fresh and aged black garlic extracts. Molecules, 21, 430.

Kim, I., Kim, J., Hwang, Y., Hwang, K., Om, A., Kim, J., \& Cho, K. (2011). The beneficial effects of aged black garlic extract on obesity and hyperlipidemia in rats fed a high-fat diet. Journal of Medicinal Plants Research., 5(14), 3159-3168.

Kim, K. J., Yu, S. H., Cho, Y. J., Pan, J. H., Cho, H. T., Kim, J. H., ... Kim, Y. J. (2017). Preparation of S-allylcysteine-enriched black garlic juice and its antidiabetic effects in streptozotocin-induced insulin-deficient mice. Journal of Agricultural and Food Chemistry, 65(2), 358-363.

Kimura, S., Tung, Y., Pan, M., Su, N., Lai, Y., \& Cheng, K. (2017). Black garlic: A critical review of its production, bioactivity, and application. Journal of Food and Drug Analysis, 62, e70.

Lata, S., Singh, S., NathTiwari, K., \& Upadhyay, R. (2014). Evaluation of the antioxidant and hepatoprotective effect of Phyllanthus fraternus against a chemotherapeutic drug cyclophosphamide. Applied Biochemistry and Biotechnology, 173, 2163-2173.

Li, X., Li, B., \& Jia, Y. (2015). The hepatoprotective effect of Haoqin Qingdan decoction against liver injury induced by a chemotherapeutic drug cyclophosphamide. Evid Based Complementary and Alternat Med., 7, 1-8.

Mahmoud, A. M., \& Al Dera, H. S. (2015). 18b-Glycyrrhetinic acid exerts protective effects against cyclophosphamide-induced hepatotoxicity: Potential role of PPARC and Nrf2 upregulation. Genes \& Nutrition, 10(6), 41.

Mahmoud, A. M., Hussein, O. E., \& Ramadan, S. A. (2013). Amelioration of cyclophosphamide-induced hepatotoxicity by the brown seaweed Turbenaria ornate. International Journal of Clinical Toxicology, 1, 9-17.

Mikaili, P., Maadirad, S., Moloudizargari, M., Aghajanshakeri, S., \& Sarahroodi, S. (2013). Therapeutic uses and pharmacological properties of garlic, shallot, and their biologically active compounds. Iran J Basic Med Sci., 16(10), 1031-1048.

Obioha, U. E., Suru, S. M., Ola-Mudathir, K. F., \& Faremi, T. Y. (2009). Hepatoprotective potentials of onion and garlic extracts on cadmium-induced oxidative damage in rats. Biological Trace Element Research, 129, 143-156.

Ohkawa, H., Ohishi, N., \& Yagi, K. (1979). Assay for lipid peroxidase in animal tissue by thiobaubituric acid reaction. Analytical Biochemistry, 95, 351-358.

Olayinka, E. T., Ore, A., Ola, O. S., \& Adeyemo, O. A. (2015). Ameliorative effect of gallic acid on cyclophosphamide-induced oxidative injury and hepatic dysfunction in rats. Medical Science, 3, 78-92.

Pedrycz, A., \& Czerny, K. (2008). Immunohistochemical study of proteins linked to apoptosis in rat fetal kidney cells following pregnancy adriamycin administration in mother. Acta Histochemica, 110, 519-523.

Public health service policy on humane care and use of laboratory animals (PHS), 2015. National Institute of Health, USA.
Rest, R. F., \& Spitznagel, J. K. (1977). Subcellular distribution of superoxide dismutases in human neutrophils. Influence of myeloperoxide measurement of superoxide dismutase activity. Biochem.J, 166(2), 145-153.

Rotruck, J. T., Pope, A. L., Ganther, H. E., Swanson, A. B., Hafeman, D. G., \& Hoekstra, W. G. (1973). Selenium: Biochemical roles as a component of glutathione peroxidase. Science, 179, 588-590.

Schumann, G., \& Klauke, R. (2003). New IFCC reference procedures for the determination of catalytic activity concentrations of five enzymes in serum: Preliminary upper reference limits obtained in hospitalized subjects. Clin Chem Acta., 327, 69-79.

Shaarawy, S. M., Tohamy, A. A., Elgendy, S. M., Abd Elmageed, Z. Y., Bahnasy, A., Mohamed, M. S., ... Matrougui, K. (2009). Protective effects of garlic and Silymarin on NDEA-induced rats hepatotoxicity. International Journal of Biological Sciences, 5(6), 549-557.

Shin, J. H., Choi, D. J., Lee, S. J., Cha, J. Y., \& Sung, N. J. (2008). Antioxidant activity of black garlic (Allium sativum L.) J. Korean Soc. Food Sci. Nutr, 37(8), 965-971.

Shin, J. H., Lee, C. W., Oh, S. J., Yun, J., Kang, M. R., Han, S., ... Kang, J. S. (2014). Hepatoprotective effect of aged black garlic extract in rodents. Toxicology Research, 30(1), 49-54.

Singh, N. P., Muller, C. H., \& Berger, R. E. (2003). Effects of age on DNA double-strand breaks and apoptosis in human sperm. Fertility and Sterility, 80, 6-1420.

Viswanatha-Swamy, A. H., Patel, U. M., Koti, B. C., Gadad, P. C., Patel, N. L., \& Thippeswamy, A. H. (2013). Cardioprotective effect of Saraca indica against cyclophosphamide induced cardiotoxicity in rats: A biochemical, electrocardiographic and histopathological study. Indian J Pharmacol., 45(1), 44-48.

Yin, S., Wei, W., Jian, F., \& Yang, N. (2013). Therapeutic applications of herbal medicines for cancer patients. Evidence-based Complementary and Alternative Medicine, 2013(1), 302426.

Zhang, C., Zeng, T., Zhao, X., Yu, L., Zhu, Z., \& Xie, K. (2012). Protective effects of garlic oil on hepatocarcinoma induced by $\mathrm{N}$-nitrosodiethylamine in rats. International Journal of Biological Sciences, 8(3), 363-374.

\section{Submit your manuscript to a SpringerOpen ${ }^{\mathcal{O}}$ journal and benefit from:}

- Convenient online submission

- Rigorous peer review

- Open access: articles freely available online

- High visibility within the field

- Retaining the copyright to your article

Submit your next manuscript at $>$ springeropen.com 